\title{
Tests de circuits intégrés au MEB à travers les couches isolantes : correction des erreurs par simulation numérique
}

\author{
H. Frémont $\left(^{*}\right)$, A. Touboul et Y. Danto \\ IXL, E.N.S.E.R.B., Université de Bordeaux I, URA 846 CNRS, 351 Cours de la Libération, F-33405 Talence \\ Cedex, France
}

(Reçu le 14 septembre 1989, révisé le 15 février 1990, accepté le $1^{\text {er }}$ mars 1990)

\begin{abstract}
Résumé. - Le contraste de potentiel créé par un faisceau d'électrons permet, grâce au phénomène de couplage capacitif, le relevé du potentiel sur des circuits intégrés passivés. L'étalement du potentiel dans les diélectriques est identifié comme une cause d'erreur particulière à ce type de mesure mais du même ordre de grandeur que les autres erreurs associées à cette technique (effet des champs locaux, bruit de grenaille des électrons secondaires...). Une simulation numérique bidimensionnelle est présentée afin de quantifier cette erreur et de corriger les valeurs mesurées.
\end{abstract}

\begin{abstract}
The voltage contrast generated by an electron beam allows the measurement of potentials by capacitive coupling mechanisms on a passivated integrated circuit. The potential spreading in the dielectric layers is identified as a typical error source related to this measurement procedure and its order of magnitude is quite similar to other errors stemming from the local fields effects, the secondary electrons shot noise ... A 2-D numerical simulation is used to evaluate this error and to correct the experimental data.
\end{abstract}

\section{Introduction.}

Le test électrique interne des circuits intégrés est un souci majeur chez les fabricants de composants électroniques, aussi bien pour le contrôle des tranches en cours de fabrication que pour l'analyse de défaillance.

Jusqu'à ces dernières années, le test par micropointes mécaniques était à la fois le plus utilisé et le plus performant. Mais la réduction toujours plus forte des motifs élémentaires et la complexité croissante des circuits intégrés font apparaître de nouvelles contraintes que les systèmes mécaniques ne peuvent surmonter (résolution spatiale et résolution temporelle insuffisantes). Aussi a-t-il été nécessaire de développer de nouvelles techniques de test ne présentant pas les défauts inhérents au contact mécanique.

Le test par faisceau d'électrons répond à ces besoins, puisqu'il est sans contact, - il n'apporte donc pas de capacité parasite-, non destructif, rapide à mettre en œuvre, et présente une résolution

$\left(^{*}\right)$ Travaux réalisés au Laboratoire du Contrôle de la Qualité, Texas Instruments, B.P. 5, 06270 VilleneuveLoubet, France. spatiale en accord avec les dimensions des motifs observés.

La couche de passivation recouvrant la surface des circuits finis crée une difficulté supplémentaire pour l'analyse. En effet, le retrait de la passivation, par voie chimique ou par plasma, est une opération délicate qui risque de dégrader le composant analysé, d'en modifier les caractéristiques électriques, ou de masquer le défaut recherché. En outre, les circuits actuels présentent plusieurs niveaux de métallisation séparés par des couches d'oxyde intermédiaire. Leur analyse nécessite donc de pouvoir mesurer, de façon qualitative et quantitative, le potentiel des pistes enterrées sous des couches isolantes.

Le phénomène de couplage capacitif créé par le faisceau d'électrons permet d'obtenir un contraste de potentiel à l'aplomb de pistes enfouies polarisées en régime dynamique. Nous avons par ailleurs montré $[1,2]$ que, si la tension d'extraction est ajustée avant chaque mesure et si l'irradiation en faisceau ponctuel est limitée à la durée de la mesure (soit environ une trentaine de secondes), cette technique, généralement utilisée pour le mode image (analyse qualitative) [3,4] peut être étendue à la mesure quantitative des variations du potentiel d'un conducteur enterré. Mais nous avons constaté expé- 
rimentalement que toutes les pistes conductrices induisent un potentiel non nul à la surface de la passivation et dans les diélectriques isolant les différents conducteurs. Si des pistes sont relativement proches du point de mesure (20 à $25 \mu \mathrm{m})$, cet " étalement du potentiel » fausse les valeurs mesurées.

Nous présentons un programme de simulation numérique bidimensionnelle, destiné à corriger ces erreurs.

\section{Approche numérique.}

Une piste conductrice polarisée par un signal continu ou variable dans le temps crée dans son entourage un potentiel non nul. Le potentiel créé dans le vide par une piste métallique superficielle est à l'origine des phénomènes de champs locaux [5-8] qui limitent la résolution en potentiel lors de la mesure au MEB. Le but de cette étude est de calculer le potentiel à la surface et dans les couches diélectriques afin de déterminer l'impact de l'étalement du potentiel et d'un couplage capacitif imparfait sur la mesure de potentiel à travers les couches isolantes. Ce phénomène (le couplage capacitif) a été observé en 1974 [9] et interprété en 1980 [10] : un contraste de potentiel, créé par un système d'imagerie électronique, peut être observé à la surface d'un isolant recouvrant une piste conductrice dont on veut connaître les variations du potentiel. Ce contraste est dû aux charges capacitivement couplées avec la surface de l'isolant: elles sont contrôlées par le faisceau et suivent les variations de potentiel du conducteur enfoui. Une étude plus approfondie a été menée dans les années $86-88[3,4,11]$ mais aucun modèle physique satisfaisant n'a encore, à notre connaissance, été proposé pour expliquer ce phénomène. Nous avons donc cherché à simuler l'effet de l'étalement du potentiel, sans considérer le couplage capacitif.

Malgré l'absence de modèle physique précis, on suppose que les phénomènes mis en jeu sont électrostatiques et que les variations de potentiel correspondent à une succession d'états d'équilibre : dans le calcul numérique, les phénomènes dynamiques sont négligés.

La configuration des circuits à analyser impose un traitement multidimensionnel. Nous avons choisi une étude bidimensionnelle, ce qui suppose que les pistes sont infiniment plus longues que larges ou épaisses (c'est le cas dans la plupart des circuits réels).

L'équation régissant la distribution de potentiel est donc une équation de Poisson :

$$
\Delta V(x, y)=\frac{\delta^{2} V}{\delta x^{2}}+\frac{\delta^{2} V}{\delta y^{2}}=-\frac{\rho(x, y)}{\varepsilon_{0} \varepsilon_{\mathrm{r}}}
$$

où $V(x, y)$ est le potentiel en un point de coordon- nées $(x, y), \rho(x, y)$ la charge par unité de volume présente dans le diélectrique, $\varepsilon_{\mathrm{r}}$ la permittivité relative du milieu, et $\varepsilon_{0}$ la permittivité du vide.

Nous supposons que le diélectrique est idéal, et donc que la charge de volume est nulle. L'expérience a prouvé que, lors d'une irradiation par le faisceau électronique, il pouvait y avoir des charges stockées à l'interface isolant-air.

Cependant, nous avons déterminé des conditions expérimentales telles que ce phénomène de charge puisse être négligé lors de la mesure du potentiel par faisceau d'électrons [12]. Dans ce cas, $\rho(x, y)=0$ dans tout l'espace, et l'équation (1) se simplifie :

$$
\Delta V(x, y)=\frac{\delta^{2} V}{\delta x^{2}}+\frac{\delta^{2} V}{\delta y^{2}}=0 .
$$

Les conditions aux limites sont imposées par les potentiels des différents conducteurs pour les frontières horizontales. Le champ sortant est pris nul sur les bords verticaux des domaines.

Cette équation de Laplace est linéarisée par la méthode des différences finies. L'espace est discrétisé, et le pas du maillage selon l'axe de déplacement du faisceau s'adapte aux dimensions réelles du faisceau électronique. La résolution de l'équation (2) est faite par colonnes (à $i$ constant) et l'équation de Laplace discrétisée prend la forme suivante :

$$
\begin{aligned}
& \frac{V(i, j-1)}{h_{j-1}\left(h_{j-1}+h_{j}\right)}+\left(\frac{1}{h_{j} h_{j-1}}+\frac{1}{h_{i} h_{i}-1}\right) V(i, j)+ \\
& +\frac{V(i, j+1)}{h_{j}\left(h_{j-1}+h_{j}\right)}=\frac{-V(i-1, j)}{h_{i-1}\left(h_{i-1}+h_{i}\right)}-\frac{V(i+1, j)}{h_{i}\left(h_{i-1}+h_{i}\right)}
\end{aligned}
$$

soit :

$$
\begin{aligned}
A(j) V(i, j-1) & +B(i, j) V(i, j)+ \\
& +C(j) V(i, j+1)=F(i, j) .
\end{aligned}
$$

Les conditions aux limites étant imposées, le système à résoudre est tridiagonal. La méthode de résolution choisie est la méthode du double balayage de Choleski [13], itérée de façon à avoir une précision convenable. On écrit le système sous la forme suivante où $V(i, j)$ est le potentiel au point $(i, j)$ :

$$
V(i, j)=E(i, j) V(i, j+1)+D(i, j)
$$

avec

$$
\begin{aligned}
E(i, j) & =\frac{-C(j)}{A(j) E(i, j-1)+B(i, j)} \\
D(i, j) & =\frac{F(i, j)-A(j) D(i-1, j)}{A(j) E(i, j-1)+B(i, j)} .
\end{aligned}
$$

Ce calcul s'applique à tous les points de la structure, à l'exception de ceux qui correspondent à 
l'interface isolant-vide. En chacun de ces points, on écrit la discontinuité de la composante normale du champ électrique :

$$
E_{0}=\varepsilon_{\mathrm{r}} E_{\mathrm{i}}
$$

où $E_{0}$ est le champ dans le vide, $\varepsilon_{\mathrm{r}}$ la constante diélectrique de l'isolant et $E_{\mathrm{i}}$ le champ dans l'isolant. L'organigramme général est donné sur la figure 1.

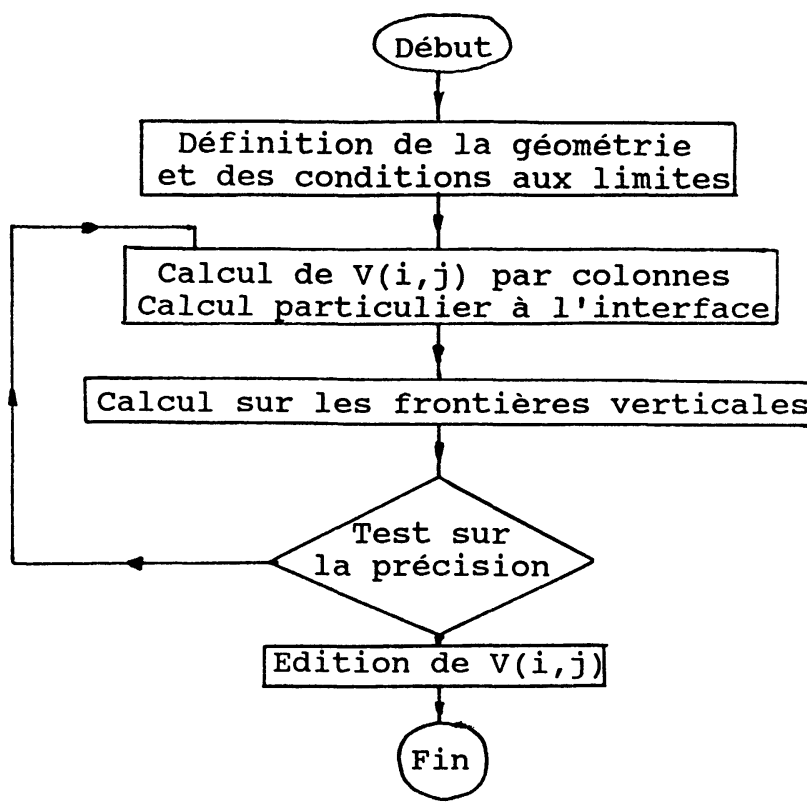

Fig. 1. - Organigramme général du calcul des potentiels.

[Flow chart for the potential calculation.]

\section{Structures de test.}

Trois structures types ont été modélisées. Les critères de choix pour la définition de ces structures ont été les suivants :

- les structures doivent être suffisamment simples pour que les résultats puissent être exploités sans risque de confusion;

- elles doivent être représentatives des configurations de circuits intégrés réels, respectant les dimensions et les positions respectives des différents conducteurs ;

- la grille d'extraction du spectromètre est prise en considération. Elle est située à $6 \mathrm{~mm}$ au-dessus du circuit.

Les figures $2 \mathrm{a}, 2 \mathrm{~b}, 2 \mathrm{c}$ présentent ces trois structures. On a indiqué les dimensions typiques utilisées pour le calcul. Ces dimensions peuvent être fixées pour chaque type de circuit étudié. Dans toute notre étude, le substrat sera relié à la masse $\left(V_{0}=0\right)$.

\section{Résultats.}

Des résultats typiques de ces simulations sont donnés sur les figures $3 a$ et $3 b$, pour deux structures différen- tes, dans un domaine limité au proche voisinage des pistes. Les calculs donnent accès au potentiel dans le vide, et par conséquent aux lignes de champ audessus du circuit. La déformation de ces lignes de champ en fonction du potentiel des différents conducteurs constitue le phénonème de champs locaux, mentionné plus haut.

Les calculs permettent également de connaître le potentiel à l'intérieur du diélectrique entre les pistes conductrices ; ceci pourrait être utilisé pour la détermination des capacités entre lignes.

Nous nous sommes plus particulièrement intéressés au potentiel de l'interface, afin de quantifier l'étalement du potentiel. Des mesures faites au MEB S200 de Cambridge Instruments associé à un système de contraste de potentiel DVCS 1500 de Lintech Instruments sur un microprocesseur Texas Instruments de technologie MOS (photographie $\mathrm{n}^{\circ}$ 1) fonctionnant à deux fréquences différentes, ont permis de valider les hypothèses du calcul (absence de charge stockée dans l'isolant, succession d'états d'équilibre). Les faibles différences que l'on peut voir sur la figure 4 entre les valeurs mesurées et les valeurs calculées sont dues au bruit propre du système de détection. Ces résultats mettent en évidence le phénomène d'étalement du potentiel; les courbes reliant le potentiel de l'interface $V_{\mathrm{i}}$ au potentiel des pistes métalliques $V_{\mathrm{m}}$ sont des droites ; or, leurs pentes varient pour cette structure du centre vers les pistes. Ainsi, $\Delta V_{\text {ic }} / \Delta V_{\mathrm{m}}$ varie de 15 à $53 \%$ selon la position du faisceau.

Les figures $5 \mathrm{a}$ et $5 \mathrm{~b}$ montrent les variations du potentiel $V_{\text {ic }}$ calculé à l'interface en fonction du potentiel $V_{\mathrm{m}}$ des grilles métalliques et en fonction du potentiel $V_{\mathrm{p}}$ de la piste enfouie respectivement, pour trois points $\mathrm{A}, \mathrm{B}, \mathrm{C}$ de la structure présentée sur la figure $2 b$. Dans les deux cas, il s'agit de droites qui ne sont pas parallèles. Lors d'un relevé de potentiel à travers une couche isolante, on ne peut avoir accès, par couplage capacitif, qu'aux variations du potentiel de la piste enfouie. On relève donc en fait un $\Delta V_{\mathrm{i}}$ qui dépend de $\Delta V_{\mathrm{p}}$. On constate que les pentes $\Delta V_{\text {ic }} / \Delta V_{\mathrm{m}}$ et $\Delta V_{\text {ic }} / \Delta V_{\mathrm{p}}$ sont dépendantes du point considéré. Ceci met en évidence l'importance du positionnement du faisceau au cours des mesures ; le point de mesure doit être choisi très précisément et, autant que possible au centre de la piste analysée pour limiter l'influence des pistes voisines.

Avant chaque relevé de potentiel, le potentiel $V_{\mathrm{e}}$ de la grille d'extraction doit être ajusté par le tracé d'une «S-curve». Les calculs numériques montrent que la pente des droites $V_{\text {ic }}\left(V_{\mathrm{m}}\right)$ est indépendante de la valeur de $V_{\mathrm{e}}$ (voir Fig. 6). Il faut cependant noter que les phénomènes de charge des isolants par la grille d'extraction étudiés dans [14] ne sont pas pris en considération ici, ce qui est justifié par nos conditions expérimentales. 


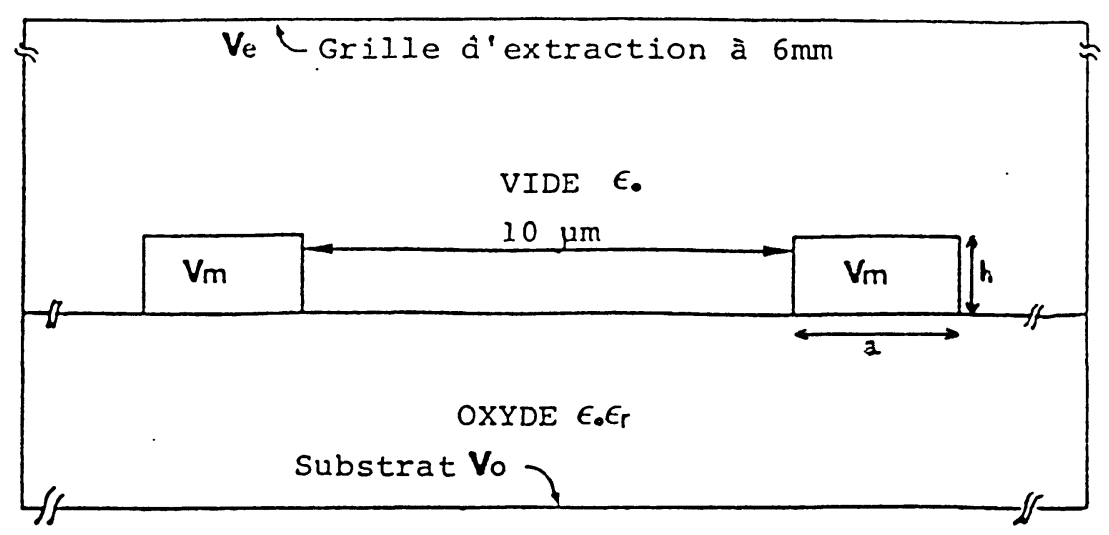

a)

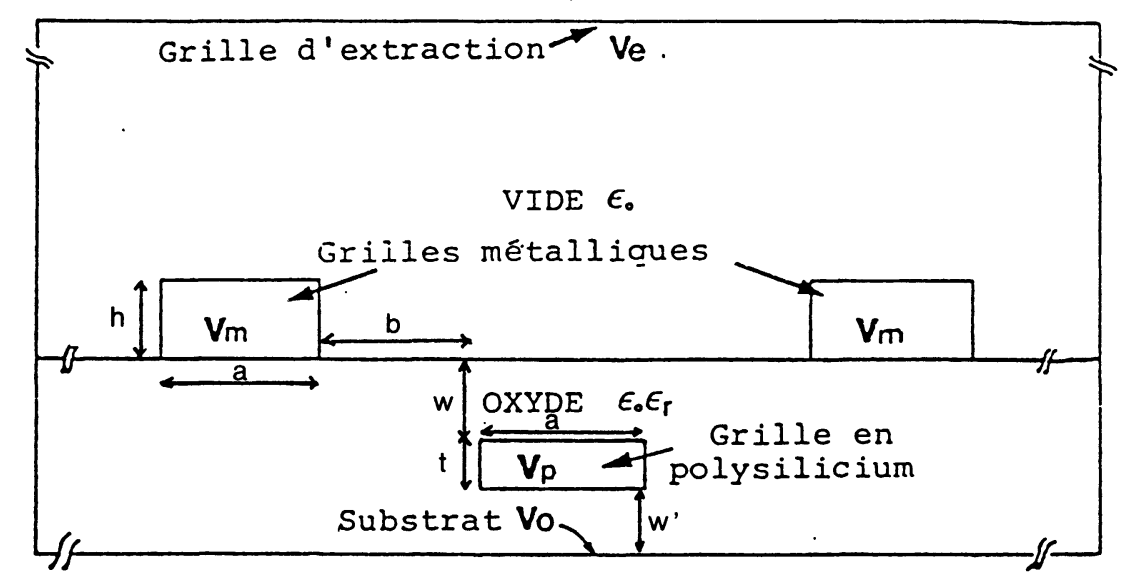

b)

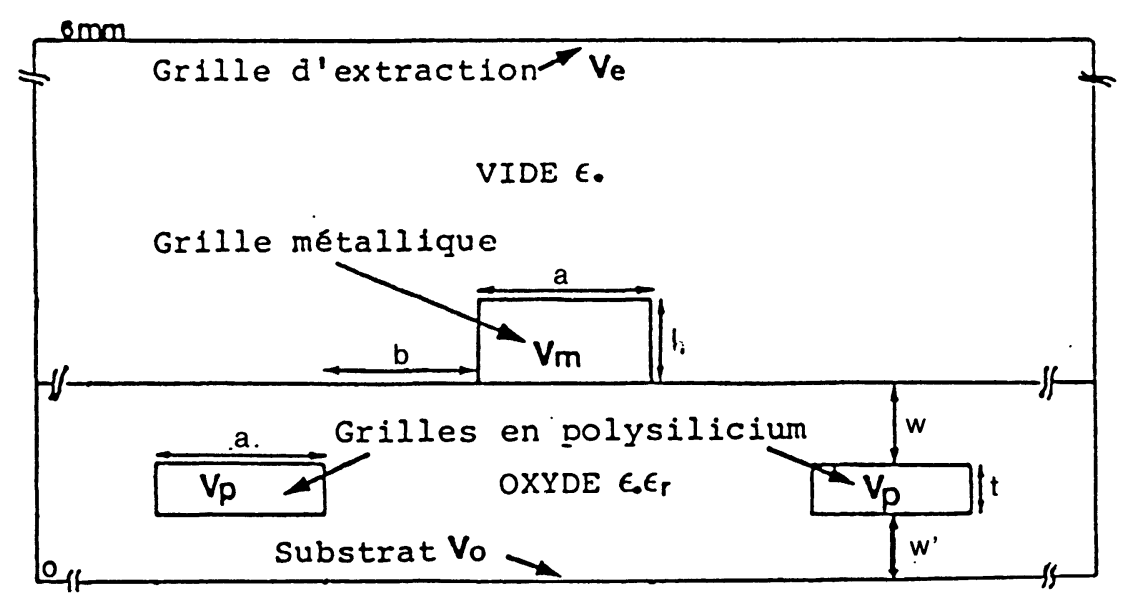

c)

Fig. 2. - Structures de test et dimensions typiques. $a=b=5 \mu \mathrm{m} ; h=w=1 \mu \mathrm{m} ; t=0,6 \mu \mathrm{m} ; W^{\prime}=0,8 \mu \mathrm{m}$.

[Test structures and typical dimensions : $a=b=5 \mu \mathrm{m} ; h=w=1 \mu \mathrm{m} ; t=0.6 \mu \mathrm{m} ; W^{\prime}=0.8 \mu \mathrm{m}$.]

Les résultats précédents se rapportent tous à des calculs réalisés pour la géométrie de la figure 2 . La figure 7 montre l'influence des dimensions sur l'écart entre la valeur calculée $V_{\text {ic }}$ et le potentiel $V_{\mathrm{p}}$ que l'on cherche à déterminer à travers la couche isolante : il apparaît que les erreurs augmentent de façon importante lorsque les dimensions des motifs élémentaires diminuent.
En particulier, il semble que la technique de test par faisceau d'électrons n'est plus utilisable pour les technologies submicroniques, car l'erreur calculée avoisine $50 \%$, quand le pas est inférieur à $2 \mu \mathrm{m}$. Afin d'évaluer l'effet de l'épaisseur d'oxyde recouvrant une piste enterrée, nous avons choisi une profondeur de $3 \mu \mathrm{m}$ au lieu de $1 \mu \mathrm{m}$. Pour un pas de $10 \mu \mathrm{m}$, comprenant une piste et un intervalle entre 

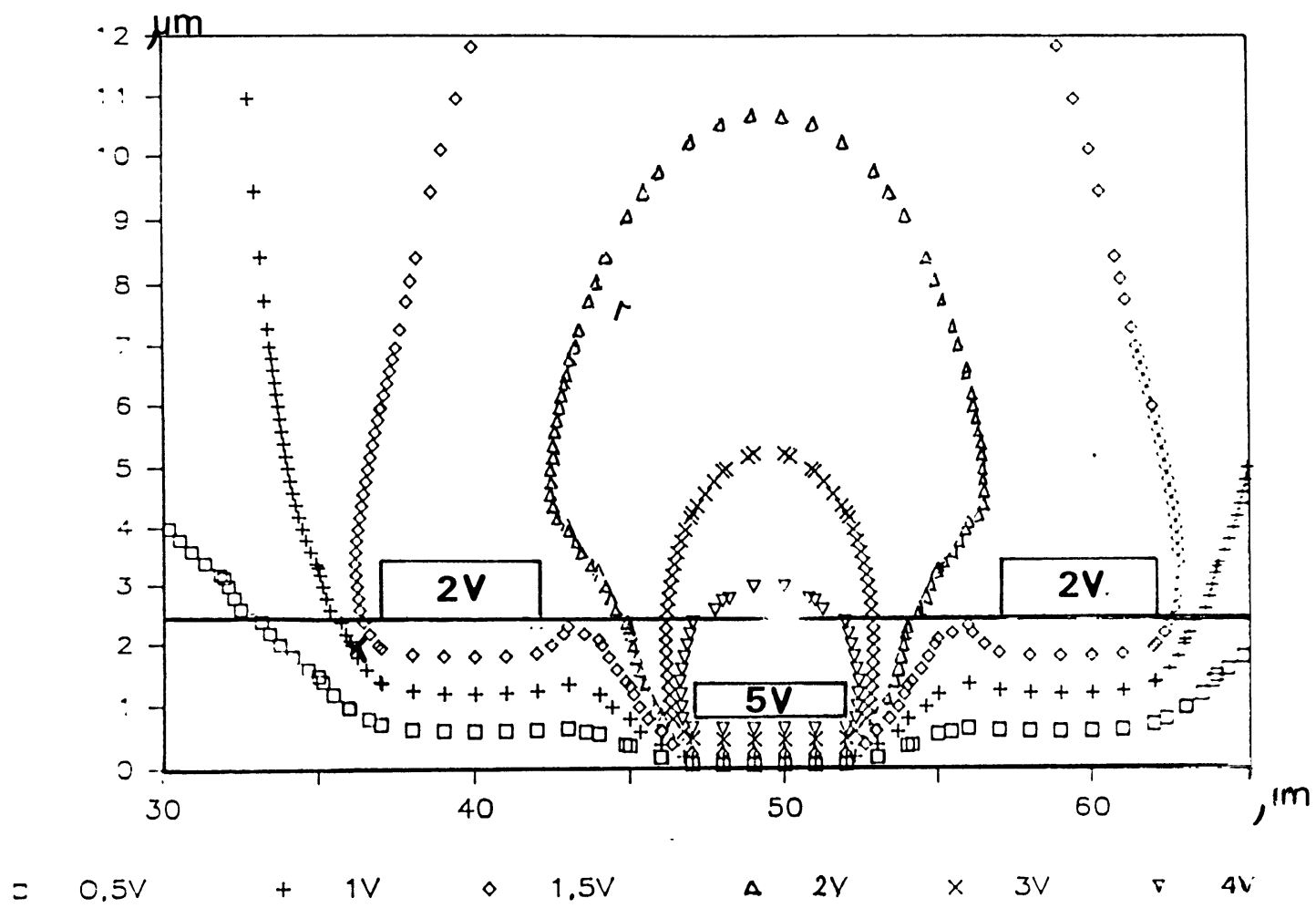

a)

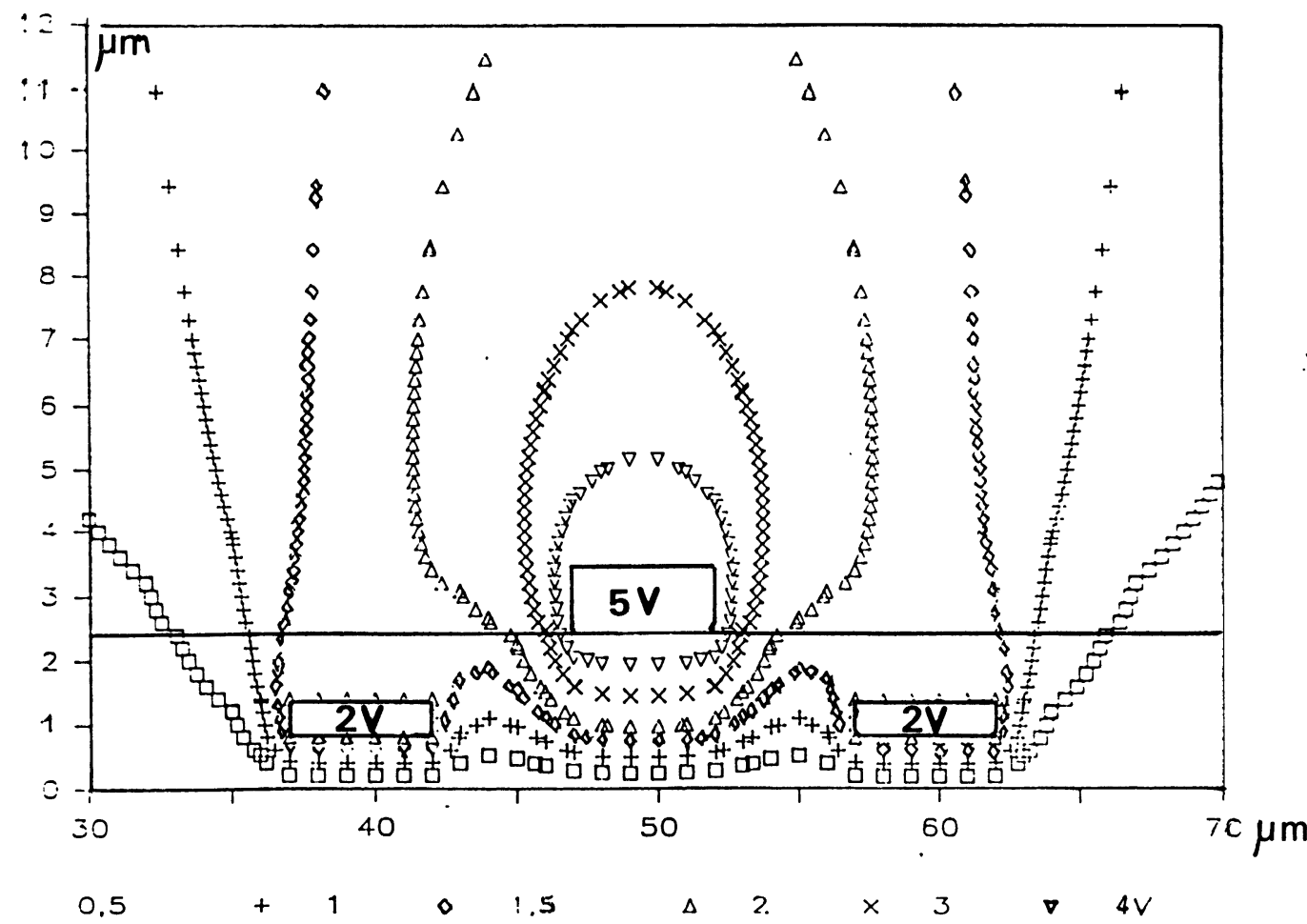

b)

Fig. 3. - a) Lignes équipotentielles pour la structure représentée sur la figure $2 \mathrm{~b}$ avec $V_{\mathrm{m}}=2 \mathrm{~V}$ et $V_{\mathrm{p}}=5 \mathrm{~V}$. b) Lignes équipotentielles pour la structure représentée sur la figure $2 \mathrm{c}$ avec $V_{\mathrm{m}}=5 \mathrm{~V}$ et $V_{\mathrm{p}}=2 \mathrm{~V}$.

(a) Equipotential lines for the figure $2 \mathrm{~b}$ lay-out with $V_{\mathrm{m}}=2 \mathrm{~V}$ and $V_{\mathrm{p}}=5 \mathrm{~V}$. b) Equipotential lines for the figure 2c layout with $V_{\mathrm{m}}=5 \mathrm{~V}$ and $V_{\mathrm{p}}=2 \mathrm{~V}$.] 


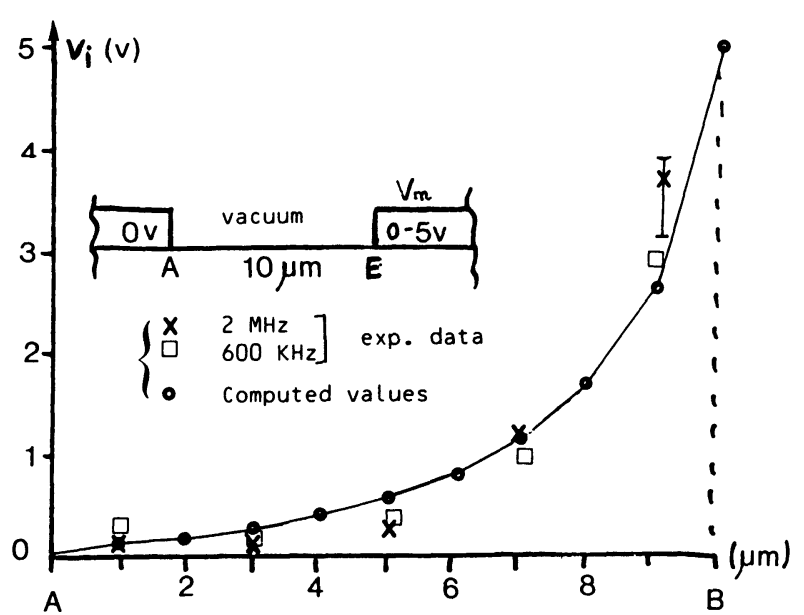

Fig. 4. - Variation du potentiel de l'interface isolant-vide entre les deux pistes métalliques (points $\mathrm{A}$ et $\mathrm{E}$ de la photo 1) comparaison des valeurs calculées (O) et des relevés expérimentaux à deux fréquences différentes $(x)$ : $2 \mathrm{MHz},(\square): 600 \mathrm{KHz}$ ).

[Interface potential variations between two metal tracks (points $\mathrm{A}$ and $\mathrm{E}$ of photo 1). Comparison of computed values $(O)$ and experimental data for two different frequencies $(x): 2 \mathrm{MHz},(\square): 600 \mathrm{KHz})$.]

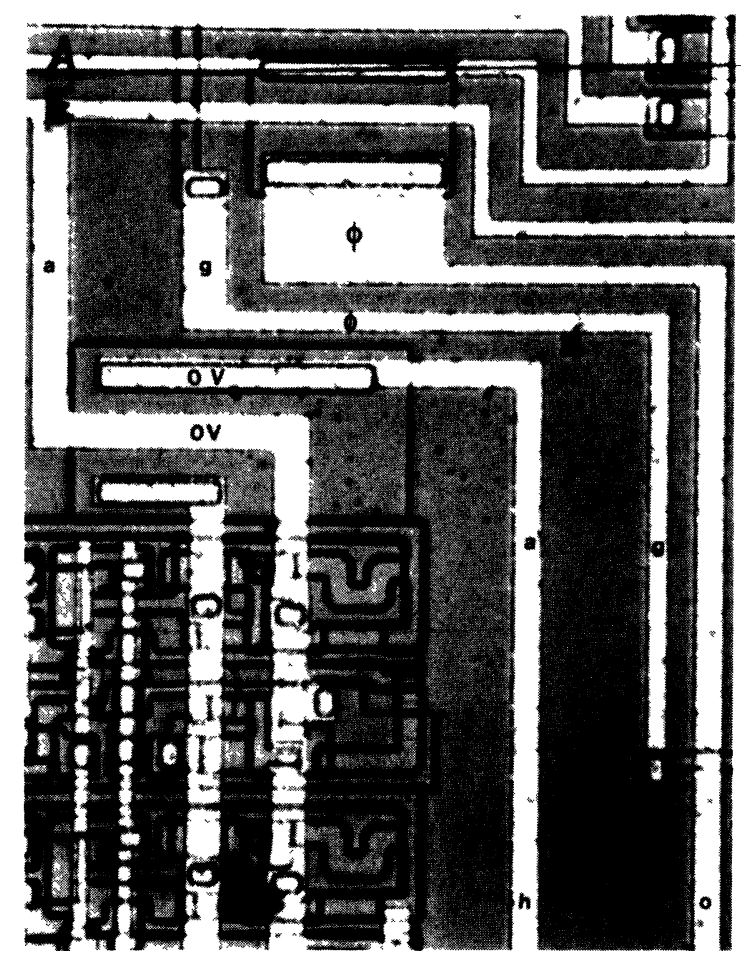

Photo 1. - Microprocesseur N-MOS de Texas Inst. testé afin d'évaluer les effets de l'étalement du potentiel (points $A$ et $\mathbf{E})$.

[View of the N-MOS microprocessor Texas Inst. under test for evaluating the potential spreading effects (E-beam impact location : A and E).]

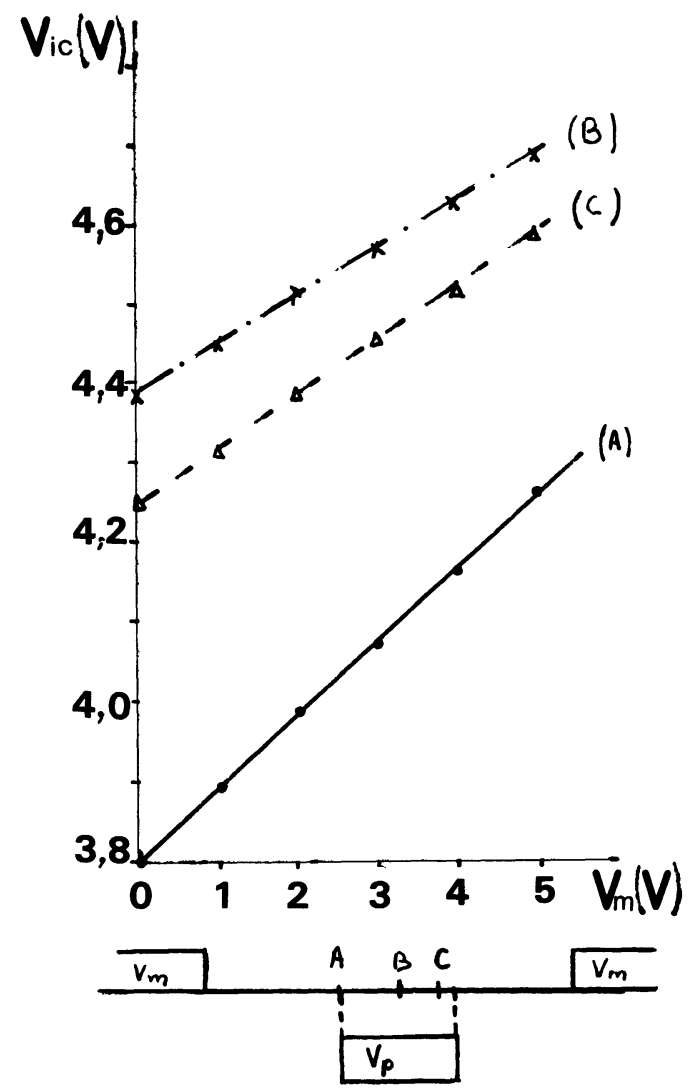

(B)

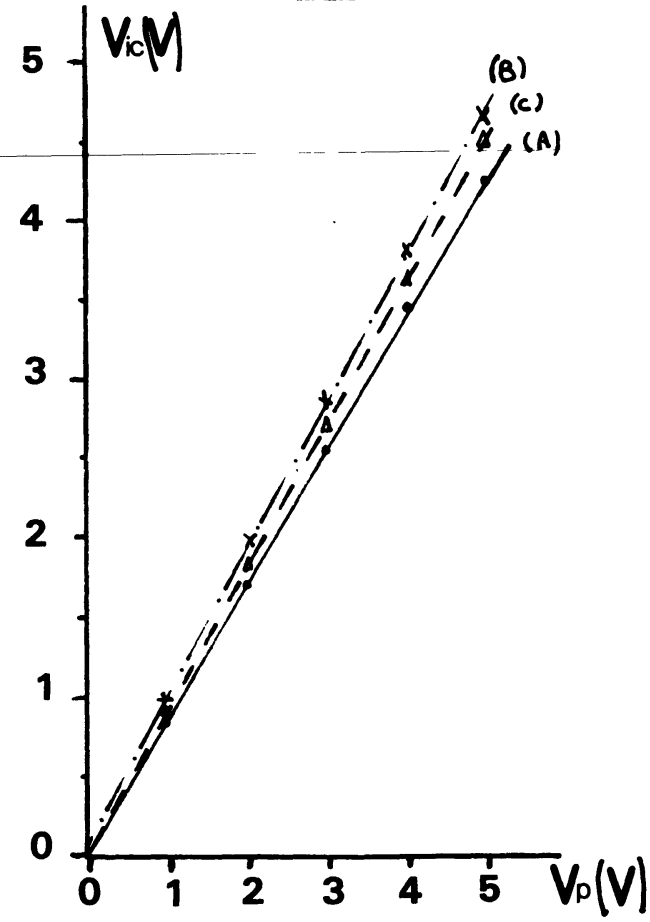

b)

Fig. 5. - Influence de la position du faisceau à l'interface isolant-vide sur la pente des droites: a) $V_{\text {ic }}\left(V_{\mathrm{m}}\right)$ pour

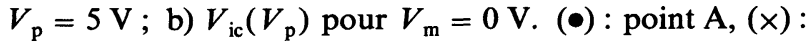
point $B,(\triangle)$ : point $C$ (ces points $A, B, C$ sont indiqués sur le Tab. I).

[Influence of the E-beam impact location at the oxidevacuum interface on the slope of the curves: a) $V_{\text {ic }} v s$. $V_{\mathrm{m}}$ for $V_{\mathrm{p}}=5 \mathrm{~V}$; b) $V_{\text {ic }}$ vs. $V_{\mathrm{p}}$ for $V_{\mathrm{m}}=0 \mathrm{~V}$. (๑) : point $\mathrm{A},(\times)$ : point $\mathrm{B},(\triangle)$ : point $\mathrm{C}$ (these points are defined in Tab. I).] 


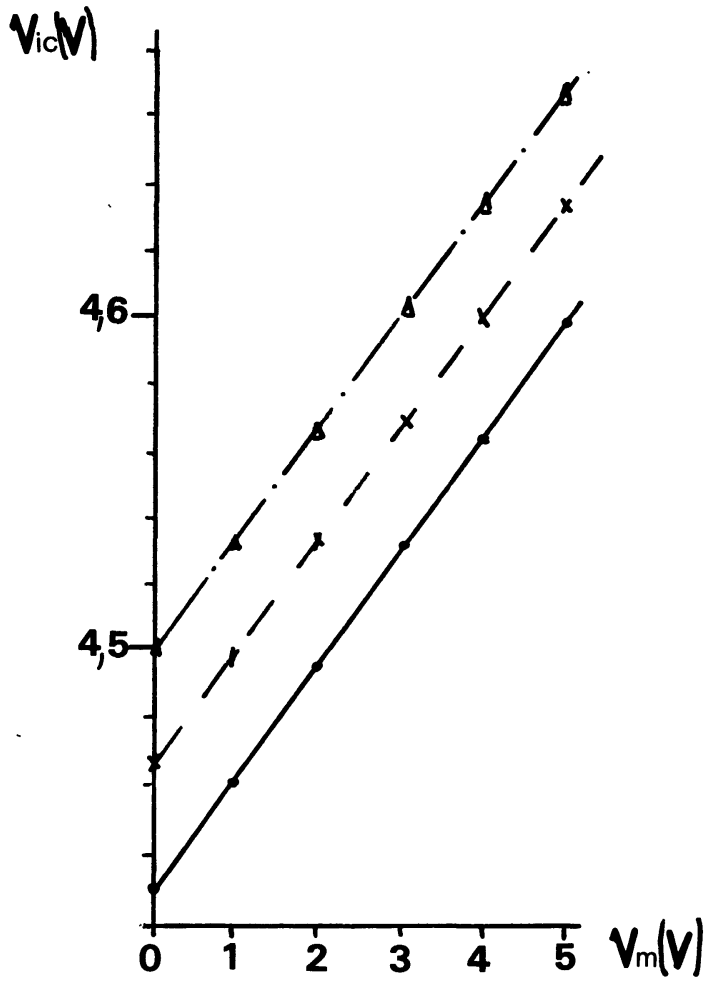

Fig. 6. - Variation de $V_{\text {ic }}$ en fonction de $V_{\mathrm{m}}$ pour trois valeurs de $V_{\mathrm{e}}$, potentiel de la grille d'extraction (structure de la Fig. 2c).

[Variation of $V_{\text {ic }} v s . V_{\mathrm{m}}$ for three values of the extraction grid potential $V_{\mathrm{e}}$ (for the Fig. 2c lay-out). $(\triangle): 600 \mathrm{~V}$, $(x): 300 \mathrm{~V},(\bullet): 0 \mathrm{~V}$.]

pistes, l'erreur maximale en cas de mauvais report par couplage capacitif est multipliée par 3 environ.

\section{Discussion.}

5.1 ANALYSE DES RÉSULTATS DES SIMULATIONS. Cette approche numérique donne accès au potentiel de l'interface, en fonction du potentiel de toutes les pistes de la structure. On peut donc écrire l'équation suivante, qui relie les variations du potentiel $V_{\text {ic }}$ calculé à l'interface aux variations de $\Delta V_{\mathrm{m}}$ et $\Delta V_{\mathrm{p}}$ :

$$
\Delta V_{\text {ic }}=f_{1}\left(\Delta V_{\mathrm{p}}, \Delta V_{\mathrm{m}}\right)
$$

Les variations de $V_{\text {ic }}$ avec $V_{\mathrm{m}}$, lorsque $V_{\mathrm{p}}$ est fixé, sont linéaires comme le montrent la figure $5 \mathrm{a}$ et les pentes de ces droites sont, en un point donné, indépendantes de $V_{\mathrm{p}}$ à $3 \%$ près (voir colonnes 3 et 4). On peut donc écire :

$$
\left(\frac{\Delta V_{\mathrm{ic}}}{\Delta V_{\mathrm{m}}}\right)_{V_{\mathrm{p}}=\mathrm{cte}}=\alpha \text { où } \alpha \text { est indépendant de } V_{\mathrm{p}} .
$$

De même, l'analyse des valeurs $V_{\text {ic }}$ en fonction de

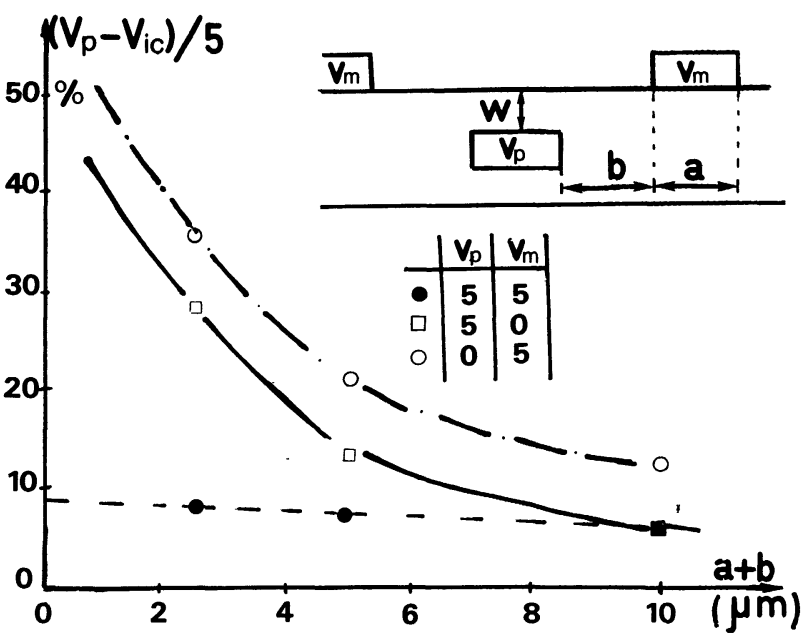

Fig. 7. - Influence de la dimension du pas élémentaire $(a+b)$ sur l'erreur normalisée $\left|V_{\mathrm{p}}-V_{\text {ic }}\right| / 5$.

[Influence of the elementary pattern $(a+b)$ dimension on the normed error $\left|V_{\mathrm{p}}-V_{\text {ic }}\right| / 5$.]

$V_{\mathrm{p}}$ (Fig. 5b) prouve que (voir Tab. I, colonnes 1 et 2) :

$$
\left(\frac{\Delta V_{\mathrm{ic}}}{\Delta V_{\mathrm{p}}}\right)_{V_{\mathrm{m}}=\text { cte }}=\beta \text { où } \beta \text { est indépendant de } V_{\mathrm{m}} .
$$

La relation (9) exprimant $\Delta V_{\text {ic }}$ s'écrit alors :

$$
\Delta V_{\mathrm{ic}}=\alpha \Delta V_{\mathrm{m}}+\beta \Delta V_{\mathrm{p}} .
$$

Les simulations numériques permettent de calculer $\alpha$ et $\beta$ pour une structure (topographie, dimensions) donnée : ce sont les pentes des droites $V_{\text {ic }}=f\left(V_{\mathrm{m}}\right)$ et $V_{\text {ic }}=f\left(V_{\mathrm{p}}\right)$ respectivement.

Il faut remarquer que le paramètre calculé $\beta$ donne accès à la valeur maximale de l'erreur $(1-\beta)$ associée à un couplage capacitif imparfait.

5.2 ANALySE DES RELEVÉS EXPÉRIMENTAUX. La mesure du potentiel de l'interface avec le faisceau électronique donne accès au potentiel de la surface en fonction du potentiel de toutes les pistes environnantes, et l'on peut écrire l'équation suivante, où $V_{\text {ir }}$ est la valeur expérimentale du potentiel relevé à l'interface :

$$
\Delta V_{\mathrm{ir}}=f_{2}\left(\Delta V_{\mathrm{p}}, \Delta V_{\mathrm{m}}\right) .
$$

On prend pour hypothèse que cette équation se met sous une forme semblable à celle de l'équation (12):

$$
\Delta V_{\mathrm{ir}}=\mu \Delta V_{\mathrm{m}}+\lambda \Delta V_{\mathrm{p}}
$$

où $\lambda$ est un coefficient inférieur ou égal à 1 , et $1-\lambda$ est égal à l'erreur en cas de mauvais report par couplage capacitif (terme «CCE » de [11]). 
Tableau I. - Valeurs calculées des coefficients $\Delta V_{\mathrm{ic}} / \Delta V_{\mathrm{p}}$ et $\Delta V_{\mathrm{ic}} / \Delta V_{\mathrm{m}}$ pour différentes valeurs des polarisations et différents points d'impact du faisceau d'électronique.

[Calculated values of the coefficients $\Delta V_{\text {ic }} / \Delta V_{\mathrm{p}}$ and $\Delta V_{\text {ic }} / \Delta V_{\mathrm{m}}$ for different values of the bias and different locations of the e-beam impact point.]

\begin{tabular}{|l|c|c|c|c|}
\cline { 2 - 5 } \multicolumn{1}{c|}{} & $\beta=\left(\frac{\Delta V_{\mathrm{ic}}}{\Delta V_{\mathrm{p}}}\right)_{V_{\mathrm{m}}=0 \mathrm{~V}}$ & $\beta=\left(\frac{\Delta V_{\mathrm{ic}}}{\Delta V_{\mathrm{p}}}\right)_{V_{\mathrm{m}}=5 \mathrm{~V}}$ & $\alpha=\left(\frac{\Delta V_{\mathrm{ic}}}{\Delta V_{\mathrm{m}}}\right)_{V_{\mathrm{p}}=0 \mathrm{~V}}$ & $\alpha=\left(\frac{(3)}{\Delta V_{\mathrm{ic}}}\right)_{V_{\mathrm{p}}=5 \mathrm{v}}$ \\
\hline $\mathrm{A}$ & $76 \%$ & $76 \%$ & $9,2 \%$ & $9,4 \%$ \\
$\mathrm{~B}$ & $88 \%$ & $88 \%$ & $5,9 \%$ & $6,0 \%$ \\
$\mathrm{C}$ & $85 \%$ & $85 \%$ & $6,7 \%$ & $6,9 \%$ \\
\hline $\mathrm{A}^{\prime}$ & $88 \%$ & $88 \%$ & $2,7 \%$ & $2,7 \%$ \\
$\mathrm{~B}^{\prime}$ & $88 \%$ & $88 \%$ & $3,6 \%$ & $3,4 \%$ \\
$\mathrm{C}^{\prime}$ & $87 \%$ & $87 \%$ & $4,8 \%$ & $4,7 \%$ \\
\hline
\end{tabular}

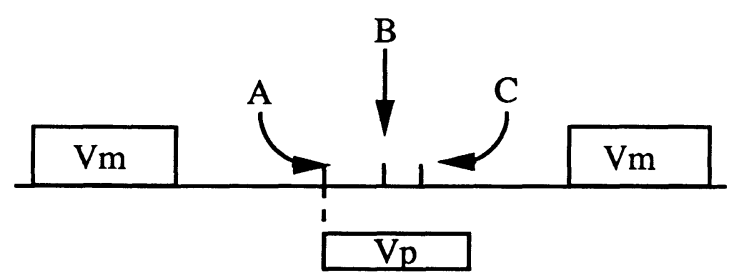

Les mesures réalisées sur trois points $\mathrm{A}, \mathrm{B}$ et $\mathrm{C}$ d'un circuit CMOS (voir photo $\mathrm{n}^{\circ}$ 2) à travers une couche de passivation de $1 \mu \mathrm{m}$ de $\mathrm{Si}_{3} \mathrm{~N}_{4}$ montrent que, pour ce type de passivation, et lorsque l'influence des pistes voisines peut être négligée, le couplage capacitif s'effectue de façon correcte [2].

Les écarts constatés sont reportés sur le tableau II. Comme ils sont très faibles, on prend $\lambda=1$.

Dans un cas plus général, on peut penser que $\lambda$ dépend de la nature et de l'épaisseur de l'isolant. Il convient donc, si possible, de vérifier expérimentalement la valeur de ce coefficient sur des zones de test du circuit.

\subsection{CORRECTION DE L'ERREUR SUR LES VALEURS} EXPÉRIMENTALES. - En l'absence de toute piste enterrée, on a vérifié expérimentalement que, en tout point de l'oxyde séparant deux pistes métalliques, les valeurs calculées sont égales aux valeurs mesurées. On écrit en comparant les relations (12) et (14) que :

$$
\mu=\alpha
$$

En cas de mauvais report par couplage capacitif, $\boldsymbol{\beta}$ représente la limite inférieure des valeurs du paramètre $\lambda$. On traduit cela par

$$
\beta<\lambda \leqslant 1 \text {. }
$$

Ainsi à partir des valeurs $\Delta V_{\text {ir }}$ et $\Delta V_{\mathrm{m}}$ relevées expérimentalement, de $\lambda$, et du coefficient numéri-
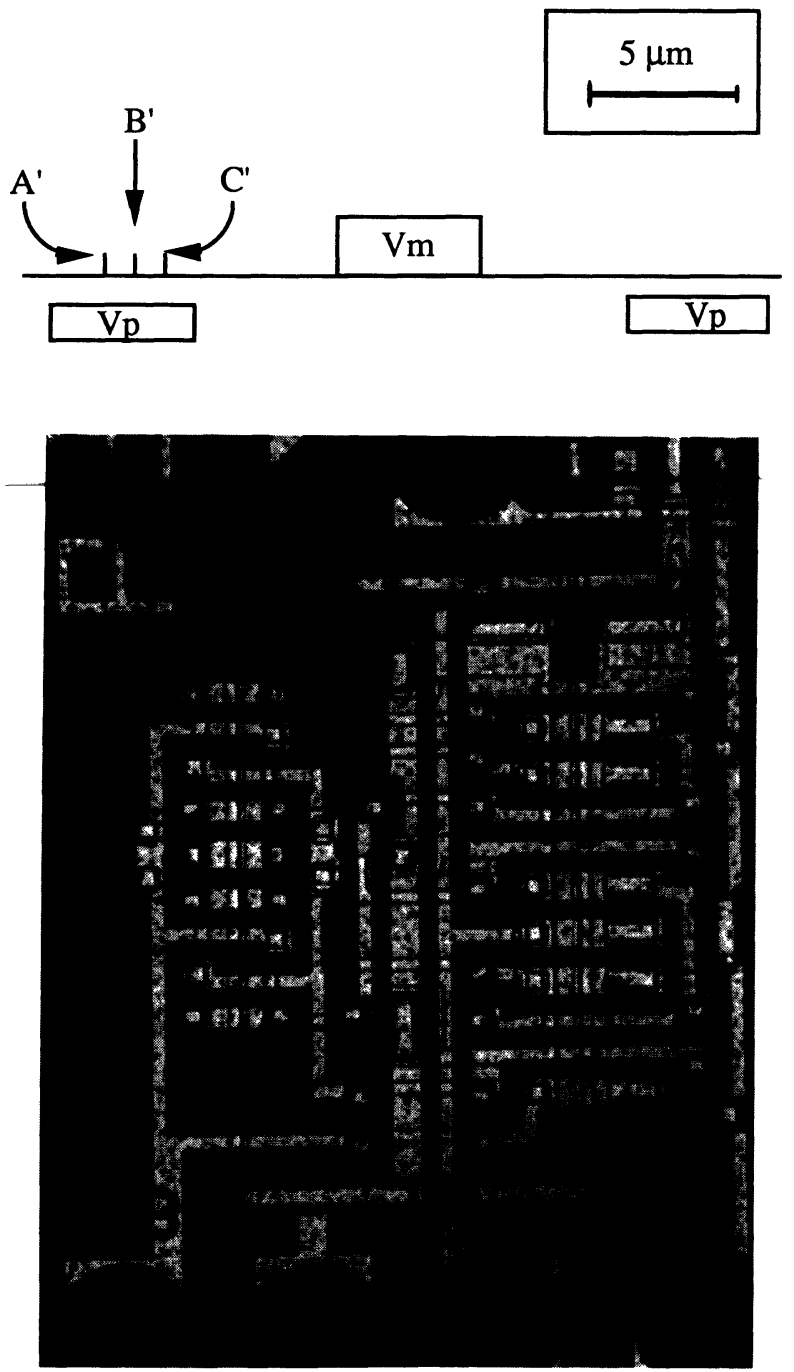

Photo 2. - Circuit CMOS de Texas Inst. utilisé afin d'évaluer la technique de mesure par couplage capacitif. Points de mesure : A, B, C.

[CMOS I.C. from Texas Inst. used to evaluate the capacitive coupling technique performances. Measurement points : A, B, C.] 
Tableau II. - Comparaison des résultats expérimentaux relatifs à des circuits passivés (colonnes de droite) et dépassivés (colonnes de gauche) décrits sur la photo 2.

[Comparison of experimental data on passivated (right columns) and depassivated (left columns) circuits shown in photo 2.]

\begin{tabular}{|c|c|c|l|l|l|r|}
\hline Point & \multicolumn{2}{|c|}{$\begin{array}{c}\text { Fréquence } \\
\text { mesurée }(\mathrm{kHz})\end{array}$} & Différence & \multicolumn{2}{c|}{$\begin{array}{c}\text { Potentiel } \\
\text { mesure (V) }\end{array}$} & Différence \\
\hline A & 23,9 & 24,0 & $0,5 \%$ & 5,0 & 4,7 & $-6 \%$ \\
B & 500 & 500 & 0 & 4,2 & 4,3 & $+2,4 \%$ \\
C & 24,6 & 24,5 & $0,1 \%$ & 4,8 & 4,7 & $-2,1 \%$ \\
\hline
\end{tabular}

que $\alpha$ de l'étalement du potentiel, on peut connaître la variation réelle $\Delta V_{\mathrm{p}}$ du potentiel de la piste enterrée qui a pour expression :

$$
\Delta V_{\mathrm{p}}=\frac{\Delta V_{\mathrm{ir}}-\alpha \Delta V_{\mathrm{m}}}{\lambda} .
$$

Dans le cas où la configuration du circuit ne permet pas d'évaluer $\lambda$, on peut avoir accès grâce aux simulations numériques, à une valeur approchée de $\Delta V_{\mathrm{p}}$ telle que :

$$
\Delta V_{\mathrm{ir}}-\alpha \Delta V_{\mathrm{m}}<\Delta V_{\mathrm{p}}<\frac{\Delta V_{\mathrm{ir}}-\alpha \Delta V_{\mathrm{m}}}{\beta} .
$$

\section{Conclusion.}

Nous avons identifié "l'étalement du potentiel» dans les diélectriques isolant les pistes conductrices comme une source d'erreurs lors du relevé de potentiel à travers les couches isolantes par la technique du couplage capacitif.

Nous avons quantifié ce phénomène par une étude numérique bidimensionnelle et comparé les résultats numériques avec des relevés expérimentaux. Pour les géométries actuelles ((largeur de piste de l'ordre de $5 \mu \mathrm{m})$, il apparaît que l'erreur due à cet étalement est du même ordre de grandeur que les autres erreurs de mesure (champs locaux, bruit de grenaille des électrons secondaires).

Cette erreur est corrigée à l'aide de coefficients obtenus d'une part grâce à l'analyse numérique, pour l'étalement du potentiel (facteur $\alpha$ ) et d'autre part avec les résultats expérimentaux, pour le couplage capacitif (facteur $\lambda$ ). Il n'existe pas de modèle physique décrivant le couplage capacitif, et les simulations numériques ne peuvent donner que la limite inférieure du paramètre $\lambda$. La détermination de $\lambda$ doit donc être faite pour chaque type de diélectrique (épaisseur, nature) et chaque condition de mesure (courant de faisceau, énergie des électrons primaires).

Pour des circuits à forte densité d'intégration, l'erreur créée par l'étalement du potentiel augmente de façon considérable. Il est donc nécessaire pour les technologies actuelles et $a$ fortiori pour les technologies submicroniques de prévoir des aires de test, dès la conception des circuits.

\section{Bibliographie}

[1] Fremont H., Touboul A., Danto Y. and Gobled D., $6^{\mathrm{e}}$ Colloque International de Fiabilité et de Maintenabilité (ADERA, Strasbourg) 1988, pp. 680-684.

[2] Fremont H., Touboul A., Danto Y. and Gobled D., $4^{\mathrm{e}}$ Conférence Internationale "Quality in Electronic Components, Prevention, Detection and Failure Analysis " (ADERA, Bordeaux) 1989, pp. 47-50.

[3] Goerlich S., Thèse de Doctorat, Université de Duisburg (RFA) 1986.

[4] Watanabe Y., FukudA Y. and JinNo T., Jpn J. Appl. Phys. 24 (1985) 1294-1297.

[5] DE JONG J. L. and ReIMER J. D., Scanning Electron Microscopy (SEM Inc., Chicago USA) 1981, pp. 933-942.

[6] Fujioka H., Nakamae K. and Ura K., Scanning
Electron Microscopy (SEM Inc., Chicago USA) 1981, pp. 323-332.

[7] Menzel E., Thèse de Doctorat, Université de Duisbourg (RFA) 1981, p. 159.

[8] WAger W. E. and Wolf E. D., J. Vac. Sci. Technol. B 4 (1986) 209-212.

[9] Crothwait D. L. and Ivy F. W., Scanning Electron Microscopy (SEM Inc., Chicago USA) 1974, pp. 935-940.

[10] Kotorman L., Scanning Electron Microscopy (SEM Inc., Chicago USA) 1980, pp. 77-80.

[11] HerrmanN K. D. and KubaleK E., Microelectron. Eng. 7 (1987) 405-415.

[12] Fremont H., Thèse de Doctorat, Université de Bordeaux I (France) 1988.

[13] Nougier J. P., Méthodes de calcul numérique (Masson, Paris) 1985, p. 27

[14] NyE P. and DiNNIS A., Scanning 7 (1985) 117-124. 